\title{
Nueva fórmula láctea para el Programa Nacional de Alimentación Complementaria: balance nitrogenado y calórico
}

\author{
Carlos Castillo D. ${ }^{1}$; Gladys Herrera A. ${ }^{1}$; Vivien Gattas $Z{ }^{1}$ \\ José Riumallo $\mathrm{S}^{1}{ }^{\text {; }}$ Sergio Jarpa $\mathrm{Y}^{2}{ }^{2}$; Francisco Alliende $\mathrm{G}^{2}{ }^{2}$
}

\section{A new modified cow's milk formula: njtrogen and energy balance studies}

\begin{abstract}
Nitrogen, fat and energy etention were imeastıped in 10 male iniants $(\bar{x}$ age $=6.8 \mathrm{mo})$ recovering from marasm ic manntrition while they were fed with either cow's milk based formula [LP) or a modified cow's milk formula (LPM). Their mean weight for age ratio was $72 \%$ and their weight tor length ratio was $95 \%$ (NCHS standards). They went through two consecutiw balance periods of 6 days each $13 \mathrm{~d}$. for odaptation and $3 \mathrm{~d}$. for urine and feces collection) beginning with either formula randomly. Both formulas had $85 \mathrm{kcal}$; d with $11 \%$ protein calories in LPM and $13 \%$ in LP. Energy intake, absorption and relative retention were silghtly greater with LPM than LP. Fat in take was greater under LP. than LP $\{5.4$ vs. $4.5 \mathrm{~g}-\mathrm{kg}-\mathrm{d}, \mathrm{p}<0.0251$, which resulted in significant differences in fat absorption (LPM 4.3 vs. LP $3 \mathrm{~g}-\mathrm{kg}-\mathrm{d}, \mathrm{P}<0.025$. Nitrogen in:ake was s:gnificantly lower under LPN than LP $\{487$ vs, $571 \mathrm{mg} \cdot \mathrm{kg} \cdot \mathrm{d}$. $0<0.025$ ) with lower ur nary nitroyen excretion tor LPM than for LF (304 vs. 417 $\mathrm{mg}-\mathrm{kg}-\mathrm{d}, \mathrm{p}<0.0011$. Apparent nitrogen retention averaged $115 \mathrm{mg} \cdot \mathrm{kg} \cdot \mathrm{d}$ for LPM and only $69 \mathrm{mg}-\mathrm{kg}-\mathrm{d} \mathrm{f}_{0 \mathrm{l}} \mathrm{LP}$ (NS). A significant correlation was observed between nitrogen intake and nitrogen retention: $r=0.701 \mathrm{y}=0.57 \times$ $-167 ; p<0.05\}$ with L!M and $r=0.77$ iv $=0.63 x-291 . p<0.011$ with LF. A correlation was a!so fourid be tween energy intake and weight gair: $r=0.55$ (NSI with LPM, and $r=0.88[y=0.26 x-26: P<0.001$ ) with LP. The modired cow's milk formula shows better nirrogen and fa: balance than the unmodified cow's milk based formula in malnourished infants recovering form marasmus.

¿Key words: nitrogen. energy, balance. cow's milk, lormulas, malnutrition!.
\end{abstract}

La leche de vaca en polvo reconstituida y adjcionada de hidratos de carbono ha sido utilizada como un eficaz. sustituto en países como el nuestro, cuando la lactancia no ha sido posible, en especial en lactantes menores de un año de edad. Esto ha ocurrido, aun cuando la leche de vaca tiene una composición que puede afectar su calidad nutricional, como relativo exceso de proteinas, insuficiencia de algunos minerales $y$ exceso de otros, con respecto a los requerimien. tos de un lactante, lo que obliga a la adición de hidratos de carbono y, o, lípidos para equilibrarla parcialmente $^{1,2}$.

El alto costo de las leches de vaca adaptadas para consumo de lactantes hunanos, ha impedido su incorporación a programas de alimentación complementaria. Sin embargo, el avance tecnológico de las industrias lecheras permite en la actualidad proponer modificaciones parciales de la leche de vaca que, sin aumentar signifi-

1. Instituto de Nutrición y Tecnología de los Alimentos (INTA), Universidad de Chile.

2. Corporacjón para la Nutrición Infantil (CONIN). cativamente sus costas, mejoren su calidad nutricional, constituyendo de esta manera mejores productos para programas de distribución de alimentos.

E1 presente trabajo forma parte de un conjunto de subproyectos destinados a evaluar la calidad nutricional, aceptabilidad y tolerancia de una keche de vaca en polvo modificada.

Sus objetivos fueron estudiar la calidad nutricional. mediante técricas de balance metabólico, de una leche de vaca en polvo modificada (LPM), en comparación con la leche de vaca en polvo corriente (LP), en lo relativo a balance nitrogenado, grasás y calorías, en lactantes desnutridos en recuperación.

\section{Material y Métodos}

Se seleccionaron 10 lactantes de sexo masculino, con desnutrición primarja, hospitalizados para su tratamiento en un centro cerrado de recuperación nutricional, perteneciente a la Corporación para la Nutrjción Infantil de Chile (CONIN). Se exigió que tuvieran entre 3 y 10 meses de edad, peso de nacimiento superior a $2.000 \mathrm{~g}$, relación peso/talla (P/T) sobre $85 \%$ 
del estándar $\mathrm{OMS}^{3}$ al momento de iniciar ef estudio, estar libres de enfermedades agudas o crónicas agregadas. Antes de ingresarlos al protocolo se solicitó autorización de los padies mediante la firma de un consentimiento escrito e informado.

Cada núño fue somerido a dos períodos sucesivos de abimentación, empleando en uno exclusivamente leche de vaca en polvo (LP) y, en el otro, leche de vaca en polvo modificada (LPM), comenzando por cual. quiera de ellas en un orden asignado al azar. $\mathrm{La}$ primera se reconstituyó al $10 \%$ con agregados de sacarosa $5 \%$, maltosa-dextrina $1 \%$ y aceite $1 \%$, mientras que 1a LPM se reconstituyó al $15 \%$, con agregados de maltosa-dextrina al 2,5\% (tabla 1).

Cada per fodo de alimentación tuvo una duración de 6 días, correspondiendo los tres prineros a adaptación a la fórmula $y$ los tres siguientes a balance. Durante los tres últimos se efectuó recolección completa de orina, mediante recolectores para 24 horas fijados a la piel con acrosol adhesivo para evitar fïtraciones $y$ también de deposiciones, usando pañales con papel filtro agregado, que habian sido pesados previamente. Al mismo tiempo se midieron culdadosamente los volúmenes ingeridos, por diferencia en el peso de las mamaderas antes y después de cada alimentación. Las pérdidas, tanto en la ingesta de fórmula, como en la recolección de orina $y$ heces, se registraron adecua. damente.

El inicio $y$ final de cada período de balance se marcó dando a ingerir al niño $100 \mathrm{mg}$ de rojo carmín y de azul brillante, respectivamente, y registrando su aparición en las heces.

Se tomaron alícuotas de alimentación, orina y fiecas, para medir el contenido de nitrógeno mediante el método de micro Kjeldhal ${ }^{4}$, grasas con el método Van de Kamers y calorías a través de bomba calorjmétrica. Además, en orina se determinó creatínina usjnaria con el objeto de controlar la exactitud de las tecolecciones y nitrógeno ureico.

Al tinal de cada período de balance se tomaron muestras de sangtc para determinar: glicemia, proteinemia, albuminemia, relación albúmina/globulina, uremia, nitrógeno ureico y fosfatasas alcalinas.

El peso corporal se controló diariamente antes de la primera alimentación; la talla se midió al inicio del estudio y al término de cada período de balance bajo condiciones estándar.

Para el análisis estadístico e interpretación de los resultados se utilizó promedio, desviación estándar y t pareado de Student para establecer diferencias de promedio. Además se efectuaron análisis de correlación simple entre variables seleccionadas.

\section{Resultados}

Los lactantes ingirieron en promedio 147,5 $\pm 22,4 \mathrm{ml} \cdot \mathrm{kg}$ - día durante el período de balance con LP y $139,4 \pm 19,4 \mathrm{ml}-\mathrm{kg}$ - día con LPM. No se observaron diferencias significativas en la ganancia ponderal entre ambos períodos $\{4,5 \pm$ 5,1 vs. $5,1 \pm 5,7 \mathrm{~g} \cdot \mathrm{kg}$-día con LP y LPM, respectivamente).

Conforme al mayor contenido proteico de la fórmula LP, la ingestión de nitrógeno fue significativamente mayor con ella que con la LPM (tabla 2). Sin embargo, la excreción fecal mostró tendencia a ser menor con la LPM y más significativamente en la excreción urinaria (417 \pm 67 vs. $304 \pm 58 \mathrm{mgN}-\mathrm{kg}$ - dia, $\mathrm{p}<0,001)$. La retención nitrogenada, con todo ello, tendió a ser mayor con la LPM, sin alcanzar significación estadística.

En lo que respecta a grasas, la ingestión fue significativamente mayor con LPM, conforme a los contenidos especificos de cada fórmula; la excreción fecal fue semejante y el balance fue mejor con LPM. En cuanto a calorias, la ingestión de éstas fue levemente mayor con LPM y no hubo diferencias significativas en la excre. ción fecal $y$ retención.

A nivel plasmático, sólo se observó mejor re. lación albümina/globulina con LPM, sin detectar-

Tabla 1

Contenido de nutrientes de la leche de vaca en polvo (LP), leche de vaca en potvo modificada (LPM) y las respectivas fórmulas preparadas

\begin{tabular}{|c|c|c|c|c|}
\hline Nutriente & $\begin{array}{c}\mathrm{LP} \\
(\mathrm{x} 100 \mathrm{~g} \text { poivo })\end{array}$ & $\begin{array}{l}\text { L.P reconstituida * } \\
\quad(x \quad 100 \mathrm{ml})\end{array}$ & $\begin{array}{c}\text { LP } \\
\text { (x 100 g polvo) }\end{array}$ & $\begin{array}{l}\text { LPM reconstituida** } \\
(\text { (x } 100 \mathrm{ml})\end{array}$ \\
\hline Proteína (g) & 27 & 2,7 & 15 & 2,2 \\
\hline Grasas (g) & 26 & 3,6 & 26 & 3,9 \\
\hline Hidratos de carbono (g) & 38 & 10,8 & 51 & 10,1 \\
\hline Acido linoletco ( $\psi_{0}$ cal total) & 1,2 & 9 & 4 & 2,3 \\
\hline Energia $(\mathrm{kcal})$ & 496 & 85,0 & 500 & 85,0 \\
\hline Sales (mg) & 5,5 & 0,6 & 4,5 & 0,8 \\
\hline
\end{tabular}

* Al $10 \%$, con agregado de sacatosa $5 \%$, malto-dextrina $2 \% y$ aceite corriente $1 \%$.

** Al $15 \%$, con agregado de malto-dextrina $2,5 \%$. 
Tabla 2

Balance nitrogenado, de grasas y calorías según ingestión de fórmulas en estudio (n ; 10 )

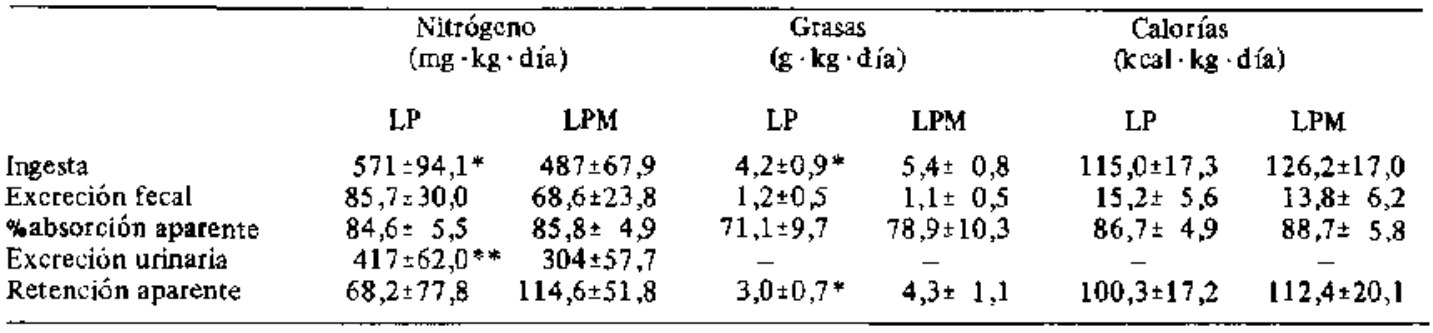

"p $<0,025 ; * * p<0,001$.

se diferencias significativas en proteinemia, albuminemia, uremia, glicemia, $\mathrm{N}$ urejco y transaminasas (tabla 3).

Se observó una correlación positiva entre nitrógeno ingerido y nitrógeno retenido con ambas fórmulas: $r=0,77(y=0,63 x-291 ; p<$ $0,01)$ con LP y $r=0,70(y=0,57 \times-167$; $\mathrm{p}<0,05$ ) con LPM (figura 1). Las mismas correlaciones fueron menos significativas al asociar $\mathrm{N}$ ingerido con $\mathrm{N}$ en orina $(r=0,58 \mathrm{y}$ $r=0,42)$. La comelación observada entre $N$ ingerido y ganancia ponderal fue de $\mathrm{r}=0,78 \mathrm{y}$ $r=0,57$ con L.P y LPM, respectivamente; el mismo análisis entre ingestión calórica y ganancia ponderal dio $r=0,88(y=0,26 x-26 ; p<$ $0,001)$ y $r=0,55(y=0,57 \times-17$; N.S. con LP y LPM, respectivamente (figura 2).

\section{Discusión}

Una de las justificaciones para el uso de le. ches adaptadas en vez de leche de vaca entera es la mayor cantidad y menor calidad de proteinas de esta última ${ }^{6}$. En contraste con los requerimientos normales, del orden de 1 a $2 \mathrm{~g}$ de proteína de alta calidad por kilogramo de peso por dia y recomendaciones que pueden ser levemente mayores para lactantes menores de un año, los lactantes del presente estudio aparecen ingiriendo $3,6 \mathrm{~g}$ proteŕna $\mathrm{kg} \cdot$ día con LP y 3,0 con LPM. Pero, además del exceso relativo en el total de proteinas, la leche de vaca tiene una proporción de proteínas de suerof caseina de 20/80. Este último es francamente diferente a la composición del patrón de la leche materna, de $60 / 40^{7}$, seguido para la LPM. Está ampliamente comprobado en muchos animales la mejor metabolización del nitrógeno proveniente de proteína del suero que de ca. seina, pero esto no ha sido evidente en estudios efectuados en el ser humano ${ }^{8}$. Además, la retención nitrogenada en lactantes de bajo peso al nacer, alimentados con proteína de leche de vaca o con leche materna, es semejante. Las diferencias pueden estar en el menor contenido

Tabla 3

Determinaciones bioquítnicas en lactantes desnutridos en recuperación según fórmulas en estudio (n : 10)

\begin{tabular}{|c|c|c|c|c|}
\hline \multirow[b]{2}{*}{ Proteinemia $(g \cdot d l)$} & \multicolumn{2}{|c|}{ Leche de vaca en polvo } & \multicolumn{2}{|c|}{$\begin{array}{l}\text { Leche de vaca en } \\
\text { polvo Modificada }\end{array}$} \\
\hline & 6,8 & $0,4 *$ & 6,5 & 0,5 \\
\hline Albuminemia $(g \cdot \mathrm{dl})$ & 4,1 & 0,2 & 4,3 & 0,4 \\
\hline Relación Alb Glob. & 1,6 & $0,3 \mathbf{a}$ & 2,0 & $0,2^{a}$ \\
\hline Uremia (mg - dl) & 26,3 & 6,2 & 25,2 & 1,1 \\
\hline N Ureico (mg $\cdot$ dl) & 12,3 & $\pm \quad 2,9$ & 11,8 & $\pm \quad 0,5$ \\
\hline Glicemin (mg $\cdot \mathrm{dl})$ & 73,5 & $\pm 17,0$ & 86,0 & $\pm 21,3$ \\
\hline Ttansaminasa GOT $(\mathbf{U} \cdot \mathbf{d l})$ & 57,1 & $\pm 32,4$ & 40,4 & $\pm 32,1$ \\
\hline
\end{tabular}

$* \bar{x} \pm \mathrm{DE} \quad \mathrm{a}: \mathrm{p}<0,01$. 


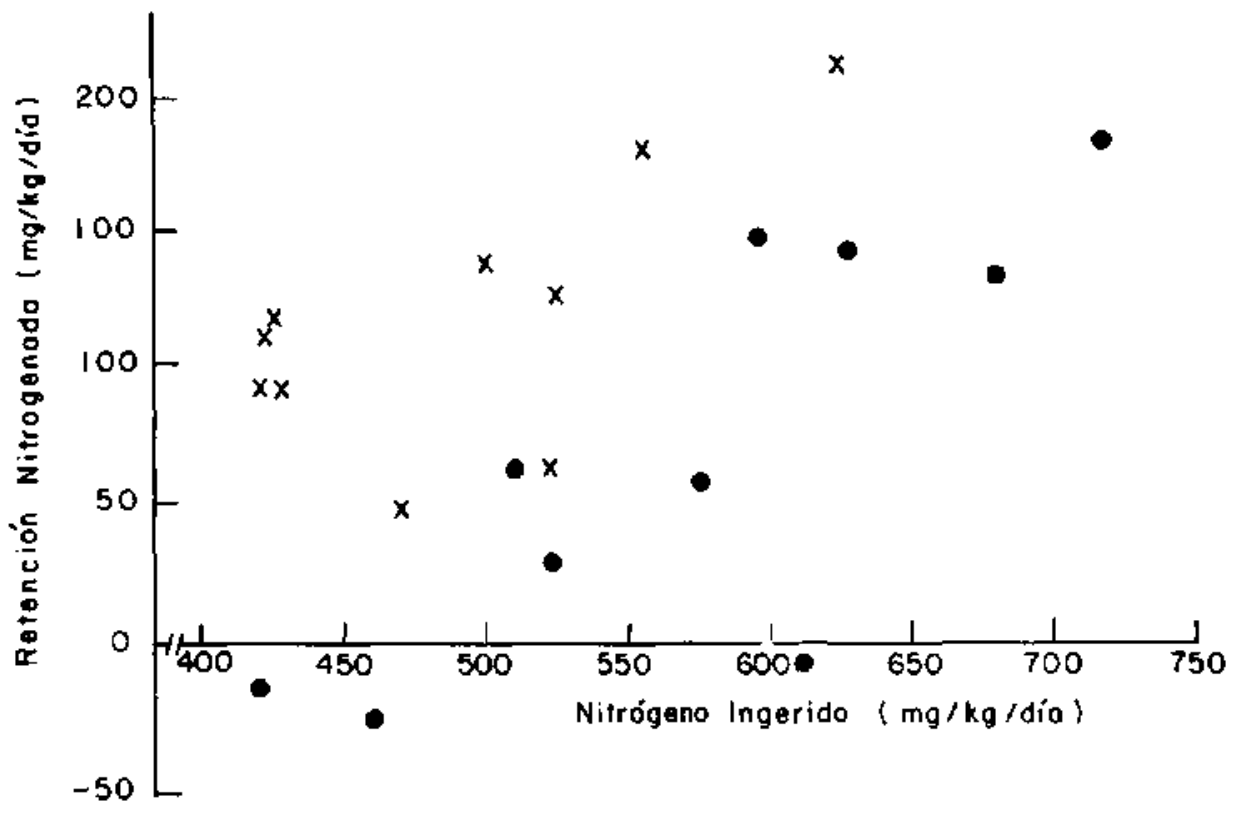

Figura 1 : Correlación entre nitrógeno ingerido y nitrógeno retenido en lactantes desnutriỏos en recuperación nutricional ( $(n=10)$, según balance con fórmula en base a lecthe de vaca (LP $\bullet$ ) o leche de vaca modificada (LPM xx). La correlación con LP fue de $\mathbf{r}=0,77 \mathrm{y}=0.63 \mathrm{x}-291$; $\mathrm{p}<0,01)$ y con LPM de $\mathrm{r}=0,70(\mathrm{y}=0,57 \mathrm{x}-167 ; \mathrm{p}<0,05)$.

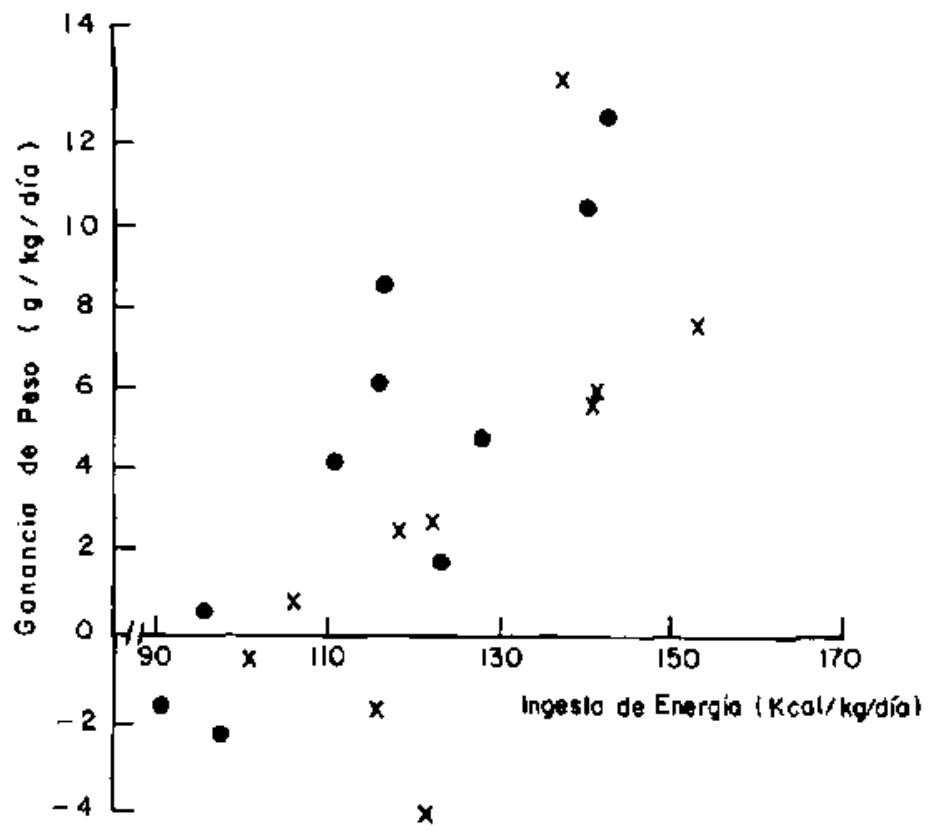

Figura 2: Carrelación entre ingesta de energía y nancia de peso en lactantes desnutridos en recuperación nutricional $(n=10)$, según balance con fómula en base a leche de vaca (LP $\bullet$ ) o leche de vaca modifjeada (LPM $x x)$. La correlación con LP fue de $I=0,88(y=0,26 x-26$; $p<0,001)$ y con LPM de $r=0,55$ (NS). 
relativo de cisteina en la caseína que en la proteína de suero y en la capacidad de esta ultima de formar grumos más pequeños y más fácilmente digeribles en el tubo gastrointestinal $^{\circledR}$. En nuestro estudio, efectuado en lactantes desnutridos en recuperación mutricional, no hubo diferencias en el porcentaje de absorción aparente de ambas proteínas; sin embargo, la retención nitrogenada tendió a ser mayor con la proteína de la LPM, como lo sugiere una significativamente menor excreción urinaria.

Los estudios de Fomon y cols. en lactantes $\operatorname{sanos}^{9}, 10$, han demostrado correlación entre las cantidades de nitrógeno ingerido y retenido, asl como también entre nitrógeno ingerido y la excreción urinaria del mismo. Este trabajo, a pesar del número relativamente pequeño de niños incorporados, muestra también una correlación semejante entre $\mathrm{N}$ ingerido $\mathrm{y}$ retenido, pero no con el $\mathrm{N}$ urinario. Además, la cantidad de $\mathrm{N}$ ingerido necesaria para mantener el balance es bastante mayor que en los estudios de Fomon. $\mathrm{La}$ asociación observada entre $\mathrm{N}$ ingerido $\mathrm{y}$ ganancia ponderal apunta también a mayor necesidad de proteinas en relación con la recuperación nutricional, requiriéndose en el caso de la leche de vaca $2,9 \mathrm{~g}$ proteina-kg-día, para $\mathrm{co}$ menzar a tener ganancia ponderal, de acuerdo con el análisis de regresión lineal (vs. $2,5 \mathrm{~g}-\mathrm{kg}$. día para LPM). Según el mismo Fomen ${ }^{10}$ en lactantes menores sanos, cuando la ingestión de $\mathbf{N}$ se acerca a los requerimientos normales, la excreción urinaria de $\mathrm{N}$ debería ser de alrededor del $91 \mathrm{mg} \cdot \mathrm{kg}$ - día. Un aumento del aporte de $N$ a cantídades como las aportadas por LPM para llegar a balance nitrogenado, aumenta la excreción de $\mathrm{N}$ a más de $130 \mathrm{mg} \cdot \mathrm{kg}$-día ${ }^{11}$. Las excreciones urinarias con las dos fórmulas estudiadas por nosotros fueron bastante superjores a las referidas (417 y $304 \mathrm{mg} \mathrm{N}$ - kg-día). Sin embargo, estos lactantes estaban en fase de recuperación nutricional. Golden y cols. ${ }^{12}$ en lactantes desnutridos estimaron que con las ingestas calóricas de nuestros niños hay una síntesis proteica de alsededor de $6 \mathrm{~g}$ - $\mathrm{kg}$-día (en base a nitrógeno retenido y nitrógeno reciclado), con retención nitrogenada aparente de cerca de $10 \mathrm{mg} \mathrm{N} \cdot \mathrm{kg}$-dia, muy semejante a las cifras encontradas por nosotros (68 y 114 mg N.kg - día para la LP Y LPM, respectiva. mente). De acuerdo con estos datos el aporte nitrogenado resulta adecuado para la condición de recuperación nutricional en que se encontra- ban nuestros pacientes, corroborado más aún por cifras normales de $\mathrm{N}$ ureico plasmático $(12,3$ y $11,8 \mathrm{mg} / \mathrm{dl}$ ). En el caso de las calorías, los resultados de la regresión muestran una necesidad de 100 cal-kg-dia para comenzar a tener aumento de peso con la misma fórmula en base a leche de vaca. La menor correlación observada para la LPM entre calorías ingeridas y ganancia de peso o balance calórico, podría estar dada por la interferencia de otros de los factores que se han modificado en la LP para obtener la fórmula modificada (contenido de ácidos grasos esenciales, vitaminas, minerales), pero también llegan a una energía de mantención cercana a $100 \mathrm{cal}$. kg-día. Spady y cols. ${ }^{\mathbf{1 3}}$, medjante calorimetria indirecta con monjtores de frecuencia cardíaca en lactantes desnutridos severos (P/T 70\%) en. contró que la energia de mantención era 81 cal-kg-día al comienzo de la recuperación nutricional, con fómulas más hipercalóricas que las usadas por nosotros (124 vs. $85 \mathrm{cal} / \mathrm{dl})$; los mismos autores cuando estudiaron los mismos lactantes en una fase más avanzada de la recuperación nutricional encontraron que la energía de mantención era $86 \mathrm{kcal} \cdot \mathrm{kg}$ - día. La diferencia con las $100 \mathrm{kcal} \cdot \mathrm{kg}$-dia, se relaciona en parte también con la mayor actividad física de los lactantes estudiados por nosotros, pues ellos estaban en fase más avanzada de recuperación nutricional y no fueron inmovilizados para efectuar los balances.

Estudios no publicados efectuados previamen. te por Uauy y cols. ${ }^{14}$, mostraban cifras mayores de energía de mantención en lactantes marásmicos en recuperación, variables entre 150 y 126, como una estimación en base a la ingesta calórico-proteica y la ganancia ponderal. En esa misma serie de estudios se encontró que la eficiencia calórica era de alrededor de $3,5 \mathrm{kcal} \cdot \mathrm{g}$ de ganancia ponderal, semejante a lo observado por nosutros, que es de $3,3 \mathrm{kcal} \cdot \mathrm{g}$ con LP y 4,9 con LPM. Esto revela alta eficiencia, si consideramos que la observada en otros estudios es de 4,5 a $5 \mathrm{kcal} \cdot \mathrm{g}^{15}$.

Con la fórmula en base a leche de vaca en polvo modificada LPM se registró mejor balance nitrogenado, dado por ingestión más adecuada para los requerimientos y menor excreción renal. El relativo exceso de aporte nitrogenado de ambas fórmulas en relación a los requerimientos normales parece apropiado para lactantes desnutridos en recuperación nutricional. También ocurtiô mejor balance de grasas que con la 
leche de vaca entera en polvo (LP), por mayor aporte de ellas y tendencia a mejor balance calúrico. Ambas fórmulas fueron capaces de provocar alta eficiencia calórica para la etapa de recuperación nutricional en que estaban los lactantes estudiados.

\section{Resumen}

Estudianos las retenciones nitrogenada, de energía y grasa en 10 lactantes (edad $\bar{x} 6,8$ meses) con desnutrición proteínico-energética en recuperación, que fueron alimentados con dos fómulas: una basada en leche de vaca entera (LP) y la segunda con una leche de vaca modifjcada (LPM). Estos nǘos tenian en promedio relación peso/edad $72 \%$ y peso/talla $95 \%$. Fueton estudiados en dos periodos consecutivos de 6 dias cada uno ( 3 para adaptación y 3 para balance) comenzando con cualquier fórmula aleatoriamente. Ambas fómulas tenían 85 $\mathrm{kcal}-\mathrm{dl}$ con $11 \%$ de calorías proteicas en LPM y $13 \%$ en LP. La ingestión, la absorción y la retención de energía fueron levemente mayores con LPM que con LP. Hubo mayor ingestión de grasa con LPM $(5,4$ vs. 4,5 g-kg-día, $\mathrm{P}<$ $0,025)$, lo que se reflejó en mayor absorción (4,3 vs. $3 \mathrm{~g} \cdot \mathrm{kg}$ - día, $\mathrm{p}<0,025)$. La ingestión de nitrógeno fue menor con LPM que con LP (487 vs. $571 \mathrm{mg}$ - $\mathrm{kg}$ - dia, p $<0,025$ ) con menor excreción urinaria de $\mathrm{N}$ (304 vs. $417 \mathrm{mg}-\mathrm{kg}$. dia, $\mathrm{p}<0,001)$. La retención aparente prome. dio de $\mathrm{N}$ fue de $115 \mathrm{mg} \cdot \mathrm{kg}$ - día para LPM $y$ $69 \mathrm{mg}-\mathrm{kg}$-día para LP (NS). Se observó una correlación significativa entre consuno y retención de nitrógeno: $r=0,70$ con LPM $(p<0,05)$ y $\mathbf{r}=0,77$ con LP $(\mathrm{p}<0,01)$. También hubo correlación entre ingesta de energia $y$ ganancia de peso: $r=0,55$ con LPM (NS) y $r=0,88$ con LP $(p<0,001)$. Concluimos que la fórmu. la basada en leche de vaca modificada muestra un mejor balance nitrogenado y de grasas que la formula en base a leche entera en lactantes des. nutridos en recuperación.

(Palabras clave: fótmula láctea, leche de vaca modificada, balance nitrogenado, balance energético, dessutrición.)

\section{Referencias}

1. Maccioni, A.: Alimentación artificial del lactante sano. Rev Chil Pediatr 1973;44:431-445.

2. $E S P G A N$ Committe on Nutrition: Guidelines on Infant Nutrition. III. Recommendations for infant feeding. Acta Paed Scand 1982 ; Suppl 302.

3. OMS: Medición del cambio del estado nutricional, Publicaciones OMS, Ginebra, 1983.

4. Assoc Offic. Agr. Chemists. Official Methods of Analysis 9th, ed. Washington, D.C.A.O.A.C. 1960.

5. Van di Kamer, J,H.: Bokkel Huiniks, U: Weijers, H.A.: A rapid method for the determination of fat in feces. J Biol Chem 177: 347, 1949.

6. Horst, C.H.; Obermannde Boer, G.L.; Kromhour, $D$.: Type of milk feeding and nutrients intake during infancy. Acta Paed Scand 1987, 76: 865.71.

7. Janas. 1.M.: Picejano, M.t.; Latch. T.F.: Indices of protein metabolism in term infants fed human milk, whey-predominant formula or cow's milk formula. Pediatrics $1985 ; 75: 775-84$.

B. Jarvenpaa, A.L.: Raiha, N.C.K.; Rassin, D.K. Gaull, G.E.: Milk protein quantity and quality in the term infant. I. Metabolic responses and effects on grow'th. Pediatrics 1982; 70: 214-20.

9. Fomon, S.J.i Thames, L.N.: Filer, L.J. Jr.; Ziegler, E.E.; Leonard, M.T.: Food consumption and growth of normal infants fed milk-based formulas. Acta Paed Scand (Suppl 223), 1971.

10. Fomon. S.J.: Proteina. En: Nutrición Infantil, Fomon, S.J. aditor, $2^{a}$ Edición, Nueva Editorial Interameticana, México D.F. 1976, pp. 112-143.

11. Axelsson, I.E.: Jakohsson, 1.; Raiha, N.C.R. Formula with reduced protein content: effects on growth and protein metabolis.n during weaning, Pediatr Res 1988; 24:297-301.

12. Golden, M.: Waterlow', I.C.: Pitou, $D$ : The relationship between dietary intake, weight change, nitrogen batance and protein turnover in man. Am J Clin Nutr 1977; 30: $1345-8$.

13. Spady, D.N.; Payne, P.R.: Picou, d.; Waterlow' $J_{1} C_{\text {: }}$ Energy balance during recovery from malnutrition. Am J Clin Nutr 1976;29: 1073-88.

14. R. Uauy: Desnutrición proteico-energética: marasmo (Comentario). En: Nutrición Clínica en la Infancia. Brunser, O.; Carraza, R.; Gracey, M.; Nichols, B.L.; Senterre, J. (editores). Raven Pres;, New York, 1985 .

15. Fomon, S.J.: Protein requirements of term infants En: Fomon, S.J.; Heird, W.C. (eds.). Energy and Protein needs during infancy. New York, Academic Press, 1986; pp. \$5-68. 